\title{
G protein-coupled receptor kinase 4 gene variants are not associated with preeclampsia in Northern Han Chinese
}

\author{
Cheng-Juan Sun and Wei-Yuan Zhang
}

Genetic variations in preeclampsia (PE) may affect PE risk. The G protein-coupled receptor kinase 4 (GRK4) gene encodes a member of the Ser/Thr protein kinase family and has been linked to both genetic and acquired hypertension. The aim of this study was to investigate the association between polymorphisms (T-rs1024323-C and T-rs1801058-C) in GRK4 and PE in Northern Han Chinese. Using a case-control design, the association between the GRK4 exon-4 T-rs1024323-C and exon-13 T-rs1801058-C polymorphisms and the risk of PE in Northern Han Chinese was assessed in 105 individuals with PE and 103 age- and area-matched normotensive controls. Genotypes were determined by allelic discrimination. The odds ratio and $95 \%$ confidence interval were estimated by binary logistic regression. No association was found between the GRK4 polymorphisms (T-rs1024323-C and T-rs1801058-C) and PE, and there was also no relationship with the severity of PE. The risk of homozygous and heterozygous variant allele carriers of the analyzed single-nucleotide polymorphisms did not differ significantly from that of the homozygous wild-type allele carriers, even after adjustment for age, body mass index, (family) history of hypertension and smoking status. The GRK4 (T-rs1024323-C and T-rs1801058-C) polymorphisms were not associated with a risk of PE in the present Northern Han Chinese study group. Thus, the GRK4 polymorphisms do not seem to have an important role in PE in this population.

Hypertension Research (2010) 33, 683-687; doi:10.1038/hr.2010.57; published online 7 May 2010

Keywords: association study; G protein-coupled receptor kinase 4; polymorphism; preeclampsia

\section{INTRODUCTION}

Preeclampsia (PE) is one of the leading causes of perinatal mortality in the world, with a great effect on the quality of life of the affected persons. Both genetic and environmental factors contribute to the pathogenesis of $\mathrm{PE}$, and gene-environmental interactions may modulate PE risk. ${ }^{1-7}$ Large epidemiological studies have shown that women with a history of early-onset severe PE, which means clinical manifestations occurring before 32 weeks of gestation, show an increased risk of cardiovascular diseases. ${ }^{8}$

The renin-angiotensin system has been shown to be important in the pathogenesis of PE. ${ }^{9}$ Dopamine can regulate blood pressure by renal and nonrenal mechanisms that involve the renin-angiotensin system. Dopamine exerts its natriuretic actions through $G$ proteincoupled receptors, which in turn are under the control of $G$ proteincoupled receptor kinases (GRKs). The amino acid changing polymorphisms of GRK4 cause hyperphosphorylation, desensitization and enhanced expression of the angiotensin II type-1 receptor. The genes encoding GRK4 localize to chromosome 4p16.3. Significant GRK4 gene heterogeneity has been shown. Two variants of the $\gamma$ isoform of GRK4, Ala142Val and Ala486Val, have been associated with GRK4 activity, resulting in increased serine phosphorylation of D1 receptors and uncoupling of the receptor from its G-protein complex. Previously, many studies of hypertension focused on GRK4 A142V and A486V were performed by polymerase chain reaction (PCR)-restriction fragment length polymorphism and direct sequencing techniques. In this study, we adopted a new method, TaqMan real-time PCR, to study the GRK4 gene variants and to analyze this relationship with PE in Northern Han Chinese (exon-4 T-rs1024323-C represents Ala142Val and exon-13 T-rs1801058-C represents A486V). The variant alleles (GRK4 Ala142Val and Ala486Val) have been associated with a higher risk of essential hypertension. ${ }^{10-12}$

A TaqMan real-time allelic discrimination system eliminates the need for post-amplification steps and is less technically demanding and more rapid than conventional PCR. This report describes the use of TaqMan real-time allelic discrimination PCR, with minor groove binding probes, for single tube detection and genotyping of SNPs. The minor groove binding molecules effectively raise the melting temperature of the oligonucleotides by binding to the minor groove of the template DNA, allowing for the use of shorter probes for real-time PCR. Thus, in an allelic discrimination assay, a single base mismatch between the template and the shorter minor groove binding probe causes more destabilization than would a single base mismatch with a 
larger probe, allowing for more accurate single-nucleotide polymorphism (SNP) detection.

Considering the functional importance of GRK4, we performed a case-control study of 105 preeclamptic and 103 normal pregnancies using two variants (T-rs1024323-C and T-rs1801058-C) of the GRK4 gene, aiming at examining the role of these polymorphisms in the development of PE in Northern Han Chinese by single locus analysis and genotype analysis.

\section{METHODS}

\section{Study population}

In this study, all DNA samples and clinical data from the participants were collected from October 2007 to April 2009 in stable residential communities in the urban district of Beijing. All participants in the study were unrelated and of Han origin without a history of intermarriage, and they were all permanent residents of Beijing. This study conformed to the ethical guidelines of the 1975 Declaration of Helsinki and was approved by the Human Studies Committee of Beijing Obstetrics and Gynecology Hospital, Capital Medical University. Informed consent was obtained from each participant. We enrolled 120 nulliparous preeclamptic subjects and 110 nulliparous healthy women from Beijing City. Of these, only 105 preeclamptic and 103 healthy pregnant subjects met the criteria. The preeclamptic group was divided into two subgroups: severe PE $(n=88)$ and mild PE $(n=17)$.

\section{Identification of PE}

The diagnosis of $\mathrm{PE}$ was performed by qualified clinicians using the criteria set by the Chinese College of Obstetrics and Gynecology (6th edition) ${ }^{13}$ for the study of hypertensive disorders complicating pregnancy. Mild PE was defined as systolic blood pressure $(\mathrm{SBP}) \geqslant 140 \mathrm{~mm} \mathrm{Hg}$ or diastolic blood pressure (DBP) $\geqslant 90 \mathrm{~mm} \mathrm{Hg}$ after 20 weeks of gestation on at least two occasions 6 or more hours apart. In addition, proteinuria was defined as $>300 \mathrm{mg}$ per $24-\mathrm{h}$ collection or persistent $1+$ protein measurement on urine dipstick. The diagnosis of severe PE required the basic features of mild PE as well as some indication of additional problems with either the mother or the baby, including: (i) very high blood pressure $(>160 \mathrm{~mm} \mathrm{Hg} \mathrm{SBP}$ or $110 \mathrm{~mm} \mathrm{Hg}$ DBP) and (ii) proteinuria $>5 \mathrm{~g}$ per 24 -h collection. Only women with the aforementioned criteria within their first and not subsequent pregnancies were included in the study. The women with normal pregnancies were chosen as healthy controls based on comparable age, Han origin and geographical location. We had access to complete clinical data for each individual, such as family history of hypertension, SBP, DBP, triglyceride level, creatinine level, birth weight and fetal growth restriction. To simplify the analyses and the interpretation of the results, subjects with a clinical history of primary liver and kidney disease, essential hypertension, diabetes mellitus, rheumatism, tumors and those who used drugs within the preceding $24 \mathrm{~h}$ were excluded from the study. None of the control subjects had a family history of PE.

\section{Specimens}

Two milliliters of whole venous blood was collected in EDTA tubes and stored at $+4{ }^{\circ} \mathrm{C}$ until use. The genomic DNA was extracted within 1 week after sampling according to the protocol of the QIAamp (QIAGEN, Chatsworth, CA, USA) DNA Blood Mini kit. DNA was diluted to $10 \mathrm{ng} \mu \mathrm{l}^{-1}$ for further study.

\section{SNP genotyping}

Specific probes for each allele (mutated and nonmutated alleles) were labeled with the fluorescence reporter dyes FAM and VIC at their $5^{\prime}$ end (sequences available on request), respectively. A nonfluorescent quencher at the $3^{\prime}$ end was used (Applied Biosystems, Foster City, CA, USA). The primers and probes were designed using Primer Express software, version 3.0 (Applied Biosystems), and sequence homogeneity was confirmed by comparison with all available sequences on the GenBank database using BLAST. Primers and probes were obtained from Applied Biosystems (Table 1). GRK4 (T-rs1024323-C and T-rs1801058-C) SNPs were genotyped using the TaqMan allelic discrimination assay and the ABI PRISM 7900 Sequence Detection System (Applied Biosystems). Briefly, the reactions were performed in $20 \mu \mathrm{l}$, comprising $10 \mu \mathrm{l}$ of TaqMan Universal PCR Master Mix $(2 \times)$, No AmpErase UNG (Applied

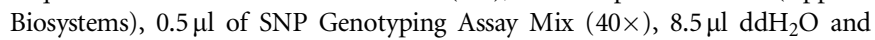
$1 \mu \mathrm{l}$ sample of DNA. All were added to TaqMan 96-well reaction plates and sealed with adhesive covers. First, the DNA was submitted to an allelic discrimination pre-read run on a PRISM 7900HT Fast real-time PCR system (Applied Biosystems). Then, the DNA was amplified under the following cycling conditions: $10 \mathrm{~min}$ at $95^{\circ} \mathrm{C}$ for AmpliTaq Gold activation and 40 cycles of $92^{\circ} \mathrm{C}$ for $15 \mathrm{~s}$ and $60^{\circ} \mathrm{C}$ for $1 \mathrm{~min}$. Moreover, a post-read run was also performed.

\section{Data analysis for determination of SNPs}

Analysis was performed using SDS software, version 2.3 (Applied Biosystems). DNA quantification was carried out by comparing the cycle threshold value (PCR cycle at which the reporter fluorescence reaches 20 s.d. above background emissions) of the samples to the cycle threshold versus plasmid quantity standard curve. Samples were considered positive if they had cycle threshold values of $\leqslant 35$ cycles. Each sample was verified visually by examining the PCR curves generated to eliminate false positives because of aberrant light emission. End-point allelic discrimination genotyping was performed by visually inspecting a plot generated from the post-PCR fluorescence read. Controls were included in each run. Within each of the two genotype groups, 20 randomly selected samples were repeated to confirm reproducibility. Each mutation detected by allelic discrimination was confirmed by direct sequencing of exon-4 T-rs1024323-C and exon-13 T-rs1801058-C of the GRK4 gene.

\section{Statistical analyses}

Markers were tested for deviation from Hardy-Weinberg equilibrium by $\chi^{2}$ goodness-of-fit tests in the control group. We used conditional logistic regression analyses, conditioned on matching factors and adjusted for potential confounders, including cigarette smoking and age factors assessed before diagnosis of PE. Genotype-based odds ratios with $95 \%$ confidence interval were calculated using individuals homozygous for the non-susceptible allele as the reference. Genotype and blood pressure multivariate analyses were assumed with multiple linear regression and analysis of covariance. For all tests, a $P$-value $<0.05$ was considered statistically significant. The logistic regression was carried out in SPSS 12.0. (SPSS, Inc., 2004, Chicago, IL, USA) Estimation of linkage disequilibrium was performed in Haploview (Haploview 3.2, Cambridge, MA, USA).

\section{RESULTS}

\section{Characteristics of participants}

The characteristics of the study subjects at diagnosis in each of the two cohorts are shown in Table 2. The cases with PE had a mean ( \pm s.d.) age of $30.36 \pm 4.99$ years compared with $29.13 \pm 3.04$ years in the

Table 1 The SNP sequence for the probes of G protein-coupled receptor kinase 4 (GRK4) genes

\begin{tabular}{|c|c|c|c|c|}
\hline NCBI SNP reference & Assay ID & Location on NCBI assembly & Celera ID & Context sequence(reverse) \\
\hline \multirow[t]{2}{*}{ rs1024323 } & C_82819 & 2975841 & hCV8281 & 5'-AAGGAGGAGAACCCTTCCAAAAAAG[C/T] -3' \\
\hline & 10_10 & & 910 & 5'-CTTTGAGGAATGTACTAGGTAAGTG-3' \\
\hline \multirow[t]{2}{*}{ rs1801058 } & C_2693 & 3008948 & hCV2693 & 5'-GTCCTGGATATCGAGCAGTTCTCGG[C/T] -3' \\
\hline & 4425_10 & & 4425 & 5'-GGTGAAAGGGATCTACCTGGACACC-3' \\
\hline
\end{tabular}

Abbreviation: SNP, single-nucleotide polymorphism. 
Table 2 Clinical characteristics of the study subjects

\begin{tabular}{lccc}
\hline & $\begin{array}{c}\text { Preeclampsia } \\
(\mathrm{n}=105)\end{array}$ & $\begin{array}{c}\text { Controls } \\
(\mathrm{n}=103)\end{array}$ & $\mathrm{P}$ \\
\hline Ages & $30.36 \pm 4.99$ & $29.13 \pm 3.04$ & $\mathrm{NS}$ \\
Husband ages & $32.92 \pm 5.20$ & $31.83 \pm 4.97$ & $\mathrm{NS}$ \\
Family history of hypertension & $30(28.6 \%)$ & $12(11.7 \%)$ & $<0.05$ \\
Smoker & $12(11.4 \%)$ & $3(2.9 \%)$ & $<0.0 .5$ \\
SBP, mm Hg & $166.05 \pm 14.38$ & $124.07 \pm 18,85$ & $<0.001$ \\
DBP, $\mathrm{mm} \mathrm{Hg}^{-2}$ & $105.94 \pm 13.09$ & $78.02 \pm 10.81$ & $<0.001$ \\
BMI, kg m${ }^{-2}$ & $23.88 \pm 4.05$ & $20.65 \pm 2.28$ & $\mathrm{NS}$ \\
TG, mmol I-1 & $3.43 \pm 1.68$ & $1.58 \pm 0.69$ & $<0.001$ \\
Cr, $\mu$ mol I $^{-1}$ & $65.22 \pm 20.16$ & $48.20 \pm 8.41$ & 0.01 \\
Birth weight, kg & $2106.77 \pm 882.72$ & $3308.13 \pm 483.15$ & $<0.001$ \\
FGR & $37(35.2 \%)$ & $2(1.94 \%)$ & $<0.05$ \\
\hline
\end{tabular}

Abbreviations: BMI, body mass index; Cr, creatinine; DBP, diastolic blood pressure; FRG, fetal growth restriction; NS, not significant; SBP, systolic blood pressure; TG, triglyceride. The family history of hypertension, SBP, DBP, TG, Cr and FRG were significantly higher in preeclampsia group than in the normal pregnancies. However, fetal birth weight was lower in the preeclamptic group than in the normal pregnancies.

normal pregnancies. The cases were also similar in terms of the husband's ages and body mass index. The family history of hypertension, SBP, DBP, triglyceride, creatinine and fetal growth restriction were significantly higher in the PE group than in the normal pregnancies, whereas the birth weight was significantly lower in the $\mathrm{PE}$ group than in the normal pregnancies.

\section{Frequency and distribution of GRK4 among PE and normal pregnancies in Chinese Northern Han}

Using the TaqMan technology, we have established an allelic discrimination assay capable of detecting two SNPs in the GRK4 gene, exon-4 T-rs1024323-C and exon-13 T-rs1801058-C. In a Chinese Northern Han population, we screened 105 cases and 103 ethnically matched normal pregnancies. The analyzed SNPs were in strong linkage disequilibrium with each other. The distribution of the alleles in the analyzed SNPs did not deviate significantly from HardyWeinberg equilibrium in the control group.

Univariate analyses indicated that the frequencies of the alanine T-rs1024323-C C allele (19.0 vs. $16.0 \%, P=0.417)$ and the alanine T-rs1801058-C C allele (59.5 vs. 50.7\%, $P=0.969)$ were not significantly different between cases and controls. There was no significant difference in the genotype distributions of the studied SNPs between the preeclamptic and control groups. The risk of homozygous and heterozygous variant allele carriers of the analyzed SNPs did not differ significantly from that of the homozygous wild-type allele carriers, even after adjustment for age, body mass index, family history of hypertension and smoking status (Table 2). No significant differences were observed in the allele and genotype frequencies between patients with mild and severe PE or between preeclamptic patients with and without fetal growth restriction. The total number of affected alleles in both rs1024323 and rs1801058 had no association between preeclamptic patients and the control group (Table 3).

\section{DISCUSSION}

PE occurs in approximately $9.4 \%$ of all pregnancies in the Chinese population. Environmental factors or reduced placental perfusion contribute to PE to some extent, but genetic factors also have a pivotal role in causing PE. In recent years, several genetic polymorphism modifications have been suggested in PE. The best approach to reducing the frequency of this complication is recognizing persons with elevated risk. In the normal adaptive mechanism of pregnancy, the renin-angiotensin system is stimulated. In contrast, vascular resistance is increased in preeclamptic patients, and they fail to develop physiologic hypervolemia. The alteration of vasoactive elements of the renin-angiotensin system could have a significant role in the pathophysiology of PE. Mutations that severely change the biochemical characteristics of synthesized proteins are not compatible with reproduction and seem to contribute significantly to PE. A novel concept is evolving that SNPs, which potentially modify gene function, might be tolerated in PE when their effects are subtle and the frequency among the population is high. Indeed, a number of such SNPs have been reported recently, and, for some of them, an association with PE functions, such as with eNOS, AGT and ACE, has been shown.

Alignments in ABI identified SNPs that were capable of differentiating the predictive allele from the remaining alleles at the locus. A strategy for the identification of PE based on the presence of specific SNPs was developed with the following allele and SNP positions: exon-4 T-rs1024323-C and exon-13 T-rs1801058-C. The TaqMan system has been shown to be rapid, sensitive and accurate for the detection of many SNPs and dispenses with post-PCR manipulations and a number of the technical problems of PCRrestriction fragment length polymorphism methods.

GRK4 is expressed in the nephron segments of the kidney, in which sodium transport is regulated by dopamine and angiotensin II. GRK4and GRK4- mediate a three- and twofold increase, respectively, in the phosphorylation of agonist-activated $\mathrm{D}(3)$ receptor, and GRK4 is crucial for its signaling in human proximal tubule cells. ${ }^{14}$ The variant of T-rs1024323-C is the allelic variant GCC to GTC that results in the amino acid substitution of alanine to valine in residue 110 , and T-rs1801058-C is the allelic variant of GTG to GCG, which results in the alanine-to-valine amino acid substitution in residue 454 . These changes are associated with a constitutive increase in GRK4 kinase activity in proximal tubular cells from humans with essential hypertension $^{15}$ (shown in Figure 1). Both the GRK4 T-rs1024323-C and T-rs1801058-C SNPs produce an alanine-to-valine amino acid change, but the effect of T-rs1024323-C on protein activity in PE is not known. Bengra et al. ${ }^{16}$ observed a significant association $(P=0.034)$ between GRK4 A486V and an Italian population of mildly hypertensive patients. Speirs et al. ${ }^{12}$ showed that the V allele of the A486V variant of GRK4- had an association with HT $(P=0.02)$. The V allele was also associated with an elevation in $\operatorname{SBP}(P=0.002)$.

After analyzing the variants, we failed to show any association of GRK4 SNPs at nucleotide T-rs1024323-C and T-rs1801058-C with PE in the Chinese Northern Han group studied. Even when the PE group was subdivided into groups with mild PE and severe PE, no association could be found. This study shows that the alterations in the relative contribution of GRK4 to the renin-angiotensin system could not affect PE risk. To our knowledge, this is the first report of a molecular epidemiological study that has suggested that genetic variation in GRK4 could not be associated with risk for PE. A comprehensive evaluation of additional SNPs in this gene is needed to estimate the importance of common haplotypes. The capability of the TaqMan 96 assay to detect a $\mathrm{T}$ variant was confirmed using a control oligonucleotide bearing a $\mathrm{T}$ in its sequence at the positions corresponding to 142 and 486 of GRK4. Even when the positive control was mixed with genomic DNA from patients, the assay gave reliable genotype determination. The result is in accordance with one study of African Americans in which there was no association between A142V and blood pressure response among African-American women. ${ }^{17}$ This is the opposite of the result found by Wang et al. ${ }^{10}$ 
Table 3 Genotypic and allelic frequencies of T-rs1024323-C and T-rs1801058-C polymorphisms of the GRK4 gene in normal pregnancies and preeclampsia

\begin{tabular}{|c|c|c|c|c|c|}
\hline Genotype & Control (\%) $(\mathrm{N}=103)$ & $P E(\%)(N=105)$ & $\chi^{2}$ & $P$ & \\
\hline \multicolumn{6}{|c|}{ T-rs1024323-C } \\
\hline $\mathrm{CC}$ & $2(1.9 \%)$ & $3(2.9 \%)$ & 0.566 & 0.748 & \\
\hline TT & $72(69.9 \%)$ & $68(64.8 \%)$ & & & \\
\hline \multicolumn{6}{|c|}{ T-rs1801058-C } \\
\hline $\mathrm{TT}$ & $15(14.6 \%)$ & $11(10.5 \%)$ & & & \\
\hline Allele & Control $(\%)(2 \mathrm{~N}=206)$ & $P E(\%)(2 \mathrm{~N}=210)$ & $\chi^{2}$ & $P$ & OR $(95 \% \mathrm{Cl})$ \\
\hline \multicolumn{6}{|c|}{ T-rs1024323-C } \\
\hline C & $33(16.0 \%)$ & 40 (19.0\%) & 0.659 & 0.417 & $0.811(0.488-1.346)$ \\
\hline $\mathrm{T}$ & $83(40.3 \%)$ & $85(40.5 \%)$ & & & \\
\hline Genotype & Control (\%) $(\mathrm{N}=25)$ & $P E(\%)(\mathrm{N}=27)$ & $\chi^{2}$ & $P$ & OR $(95 \% \mathrm{Cl})$ \\
\hline \multicolumn{6}{|c|}{ Affected genotype in both rs 1024323 and rs 1801058} \\
\hline $\mathrm{C} / \mathrm{T}$ & $14(56.0 \%)$ & $20(74.1 \%)$ & 1.874 & 0.245 & $0.674(0.391-1.162)$ \\
\hline $\mathrm{TT}$ & $11(44.0 \%)$ & $7(25.9 \%)$ & & & \\
\hline Allele & Control $(\%)(2 \mathrm{~N}=50)$ & $P E(\%)(2 \mathrm{~N}=54)$ & $\chi^{2}$ & P & OR $(95 \% \mathrm{Cl})$ \\
\hline \multicolumn{6}{|c|}{ Affected allele in both rs 1024323 and rs 1801058} \\
\hline C & $14(28 \%)$ & $20(37 \%)$ & 0.326 & 0.404 & $0.661(0.289-1.514)$ \\
\hline
\end{tabular}

Abbreviations: $\mathrm{Cl}$, confidence interval; GRK4, G protein-coupled receptor kinase 4; OR, odds ratio; PE, preeclampsia.

No significant differences were observed in the allele and genotype frequencies between patients with mild and severe PE or between preeclamptic patients with and without fetal growth restriction. The total number of affected alleles in both rs1024323 and rs1801058 have no association between preeclamptic patients and control group.

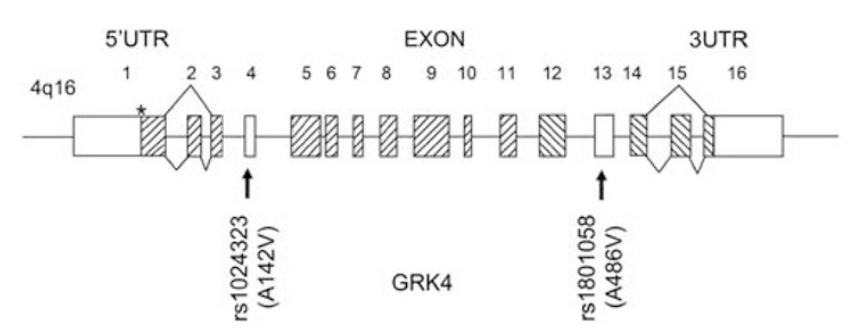

Figure 1 Schematic representation of the GRK4 gene and positions of the measured SNPs. Hatched boxes indicate the exons of GRK4. The sketch in exon 4 and 13 shows the localization of the GRK4 (T-rs1024323-C and T-rs1801058-C) SNP, which are described in open boxes.

in the Chinese Northern Han population. Although Wang et al. and Bhatnagar et al. analyzed T-rs1024323-C and T-rs1801058-C in hypertension, we selected subjects with PE. In addition, we observed an association of the $\mathrm{T}$ allele of the T-rs1024323-C variant with an elevation in DBP; this is also in agreement with the result found by Speirs et al. ${ }^{12}$. These controversial results may be due to populationspecific differences in the extent of linkage disequilibrium between rs1024323 and a putative functional SNP, either in the GRK4 gene or in nearby regions. Thus, further studies covering more genomic regions than this study may help to test this hypothesis. Different sample sizes may also contribute to discrepant results. Compared with the study of $168 \mathrm{HTs}$ and 312 NTs (Speirs et al. ${ }^{12}$ ), our sample sizes are considerably smaller.

Several reasons may explain these inconsistent findings, including allelic heterogeneity, true variation in disease association between populations, modifying genetic and/or environmental factors, or statistically underpowered small sample sizes. The most parsimonious explanation for the lack of replication of initial GRK4 association results in our study would be a variation in disease association with a relatively small sample size.

Although this is a negative study, it is important because it highlights the fact that previously reported associations between polymorphisms in GRK4 and either hypertension and/or salt sensitivity may be restricted to essential hypertension and failure to eliminate sodium after a sodium challenge. As hypertension and salt sensitivity are primarily mediated in the kidney, one would have expected GRK4 to be unrelated to the primary cause of PE, which is thought to originate at the maternal-fetal interface and to be immunologic in nature. Thus, this study is useful in confirming that GRK4 is probably not related to the elevation in blood pressure found in PE.

In summary, the GRK4 T-rs1024323-C and T-rs1801058-C polymorphisms were not associated with risk of $\mathrm{PE}$ in the present Chinese 
study group, indicating that this polymorphism does not have an important role in PE in the Northern Chinese population.

\section{CONFLICT OF INTEREST}

All authors have made a substantial contribution to the text submitted for publication, and all have read and approved the final paper. No author has substantial direct or indirect commercial or financial incentive associated with the publishing of this paper.

\section{ACKNOWLEDGEMENTS}

We thank the participants of the study. Thanks are also extended to two anonymous reviewers for their very thoughtful comments, criticisms and suggestions for revision. Wei-Yuan Zhang, MD was the guarantor of the article. This study was supported by talent project of the Beijing Health Bureau (Grant No. 2009-2-11).

1 Molvarec A, Kalabay L, Derzsy Z, Szarka A, Halmos A, Stenczer B, Arnaud P, Karádi I, Prohászka Z, Rigó Jr J. Preeclampsia is associated with decreased serum alpha(2)-HS glycoprotein (fetuin-A) concentration. Hypertens Res 2009; 32: 665-669.

2 de Lima TH, Sass N, Mattar R, Moron AF, Torloni MR, Franchim CS, Daher S. Cytokine gene polymorphisms in preeclampsia and eclampsia. Hypertens Res 2009; 32: 565-569.

3 Hirashima C, Ohkuchi A, Matsubara S, Suzuki H, Takahashi K, Usui R, Suzuki M. Alteration of serum soluble endoglin levels after the onset of preeclampsia is more pronounced in women with early-onset. Hypertens Res 2008; 31: 1541-1548.

4 Zafarmand MH, Franx A, Sabour S, van der Schouw YT, Grobbee DE, de Leeuw PW, Bots $M L$. The M235T variant of the angiotensinogen gene is related to development of selfreported hypertension during pregnancy: the Prospect-EPIC cohort study. Hypertens Res 2008; 31: 1299-1305.

5 Canto P, Canto-Cetina T, Juárez-Velázquez R, Rosas-Vargas $H$, Rangel-Villalobos $H$, Canizales-Quinteros S, Velázquez-Wong AC, Villarreal-Molina MT, Fernández G, CoralVázquez R. Methylenetetrahydrofolate reductase C677T and glutathione S-transferase P1 A313G are associated with a reduced risk of preeclampsia in Maya-Mestizo women. Hypertens Res 2008; 31: 1015-1019.
6 Molvarec A, Jermendy A, Kovács M, Prohászka Z, Rigó Jr J. Toll-like receptor 4 gene polymorphisms and preeclampsia: lack of association in a Caucasian population. Hypertens Res 2008; 31: 859-864.

7 Molvarec A, Vér A, Fekete A, Rosta K, Derzbach L, Derzsy Z, Karádi I, Rigó Jr J. Association between estrogen receptor alpha (ESR1) gene polymorphisms and severe preeclampsia. Hypertens Res 2007; 30: 205-211.

8 Bellamy L, Casas JP, Hingorani AD, Williamsm DJ. Pre-eclampsia and risk of cardiovascular disease and cancer in later life: systematic review and meta-analysis. BMJ 2007; 335: 974.

9 Zhang G, Wang H, Wang F, Yu L, Yang X, Meng J, Qin W, Wu G, Li J, Yang A, Zhou Y, Zhang R. Gene polymorphisms of the renin-angiotensin-aldosterone system and angiotensin II type 1-receptor activating antibodies in renal rejection. Tohoku J Exp Med 2007; 213: 203-214.

10 Wang Y, Li B, Zhao W, Liu P, Zhao Q, Chen S, Li H, Gu D. Association study of G proteincoupled receptor kinase 4 gene variants with essential hypertension in northern Han Chinese. Ann Hum Genet 2006; 70: 778-783.

11 Felder RA, Sanada H, Xu J, Yu PY, Wang Z, Watanabe H, Asico LD, Wang W, Zheng S, Yamaguchi I, Williams S, Gainer J, Brown NJ, Hazen-Martin D, Wong LJ, Robillard JE, Carey RM, Eisner GM, Jose PA. G protein-coupled receptor kinase 4 gene variants in human essential hypertension. Proc Natl Acad Sci USA 2002; 99. 3872-3877.

12 Speirs HJ, Katyk K, Kumar NN, Benjafield AV, Wang WY, Morris BJ. Association of Gprotein-coupled receptor kinase 4 haplotypes, but not HSD3B1 or PTP1B polymorphisms, with essential hypertension. J Hypertens 2004; 22: 931-936.

13 Gou W. Hypertensive disorder complicating pregnancy. In: Jie Le (ed). The Chinese College of Obstetrics and Gynecology, 6th edn. People's Medical Publishing House, China, 2004, pp. 97-106.

14 Villar VA, Jones JE, Armando I, Palmes-Saloma C, Yu P, Pascua AM, Keever L, Arnaldo FB, Wang Z, Luo Y, Felder RA, Jose PA. G protein-coupled receptor kinase 4 (GRK4) regulates the phosphorylation and function of the dopamine D3 receptor. $\mathrm{J}$ Biol Chem 2009; 284: 21425-21434.

15 Jose PA, Eisner GM, Felder RA. Dopamine and the kidney: a role in hypertension? Curr Opin Nephrol Hypertens 2003; 12: 189-194.

16 Bengra C, Mifflin TE, Khripin Y, Manunta P, Williams SM, Jose PA, Felder RA. Genotyping of essential hypertension single-nucleotide polymorphisms by a homogeneous PCR method with universal energy transfer primers. Clin Chem 2002; 48: 2131-2140.

17 Bhatnagar V, O'Connor DT, Brophy VH, Schork NJ, Richard E, Salem RM, Nievergelt CM, Bakris GL, Middleton JP, Norris KC, Wright J, Hiremath L, Contreras G, Appel LJ, Lipkowitz MS, AASK Study Investigators. G-protein-coupled receptor kinase 4 polymorphisms and blood pressure response to metoprolol among African Americans: sexspecificity and interactions. Am J Hypertens 2009; 22: 332-338. 